\title{
Size-selective diffusion in nanoporous alumina membranes for a glucose affinity sensor
}

\author{
Christophe Boss ${ }^{\mathrm{a}, *}$, Eric Meurville ${ }^{\mathrm{a}}$, Jean-Michel Sallese ${ }^{\mathrm{b}}$, Peter Ryser ${ }^{\mathrm{a}}$ \\ a Laboratory of Microengineering for Manufacturing, Ecole Polytechnique Fédérale de Lausanne, Station 17, 1015 Lausanne, Switzerland \\ b Institute of Electrical Engineering, Ecole Polytechnique Fédérale de Lausanne, Station 11, 1015 Lausanne, Switzerland
}

\section{A R T I C L E I N F O}

Article history:

Received 16 November 2011

Received in revised form 1 February 2012

Accepted 2 February 2012

Available online 11 February 2012

Keywords:

Nanoporous membrane

Alumina membrane

Biosensor membrane

Affinity sensor

Glucose sensor

\begin{abstract}
A B S T R A C T
The retention, diffusion and structural properties of $2-4 \mathrm{~nm}$ nanoporous alumina membranes were investigated in view of their integration as size-selective interface in a glucose sensor. These membranes exhibit remarkable glucose diffusion properties with only a 5-fold reduction compared to free diffusion in water. The retention of the glucose-binding protein (Concanavalin A), which is characterized by a hydrodynamic radius of only $3.3 \mathrm{~nm}$, was almost complete during at least 35 days. This high selectivity was also confirmed by SEM picture analysis showing a highly uniform pore size distribution. Finally, the glucose sensor including a nanoporous membrane as size-selective interface was able to measure glucose levels in physiological solution during 25 days, which confirms that annealed alumina membranes are well suited for size-selective interface of biosensors.
\end{abstract}

(c) 2012 Elsevier B.V. All rights reserved.

\section{Introduction}

Nanoporous membranes are often required for biosensors operating in complex biological fluids such as blood or plasma. Their role is to exclude large biomolecules from the blood which could interfere with the measurements, as well as to minimize biofouling. For the sensor to work properly, the membrane has to be biocompatible, stable in aqueous environment, and allow fast diffusion in the sensor [1]. On the other hand, nanoporous membranes can also be used to confine large sensing molecules provided they exhibit a well defined pore size distribution. Various types of nanoporous membranes have been investigated for biosensors applications, including polymeric membranes [2], micromachined silicon membranes [3] and anodic alumina membranes [4]. With their good structural stability and intrinsic biocompatibility, nanoporous alumina membranes are typically well suited for biosensor size-selective interface. These membranes obtained under specific anodization conditions exhibit highly ordered arrays of well defined pore diameters. Moreover, alumina membranes may be annealed, giving them an excellent chemical resistance and long term stability in aqueous solution. Nanoporous alumina membranes have been used for various applications of nanofiltration in biotechnology [5], for molecular separation in medical devices [6,7], as well as for dialysis in biosensors [8]. Recently,

\footnotetext{
* Corresponding author. Tel.: +41 216937780; fax: +41 216933891.

E-mail address: christophe.boss@epfl.ch (C. Boss).
}

membranes with pore sizes as small as $2-4 \mathrm{~nm}$ have been commercially available (Synkera Technologies, Inc.), thus foreseeing promising application for biosensors. Such membranes are characterized by a bilayer structure including a very thin active layer $(0.5-1.5 \mu \mathrm{m})$ and a support layer with larger pores $(150-200 \mathrm{~nm})$ ensuring rigid membranes with good diffusivity properties.

For fifty years, considerable efforts have been devoted to developing glucose sensors which could be used for continuous monitoring of diabetic patients [9]. Amongst others, one approach has been the affinity sensing technique, which is based on the binding of glucose with a carbohydrate-specific molecule $[10,11]$. We recently reported promising results for an affinity based sensor relying on the detection of the viscosity variation of a sensitive fluid with the glucose concentration [12]. The sensitive fluid principle relies on the competitive binding of glucose and dextran with a glucose-specific binding protein, Concanavalin A (ConA). Basically, the sensor is filled with the sensitive fluid which is confined by a nanoporous alumina membrane used as size-selective interface (Fig. 1). The sensitive fluid viscosity is detected by inducing a flow in a microchannel upon piezoelectric diaphragm actuation. The nanoporous membrane is a key element since it has to confine ConA molecules whilst allowing fast glucose permeation. In this paper, we report experimental results on diffusion and retention of annealed nanoporous alumina membranes as well as structural properties. ConA retention and glucose diffusion are investigated using respectively ultraviolet absorption spectrometry and refractometry. The pore size distribution and the porosity is also characterized using scanning electron microscopy. 


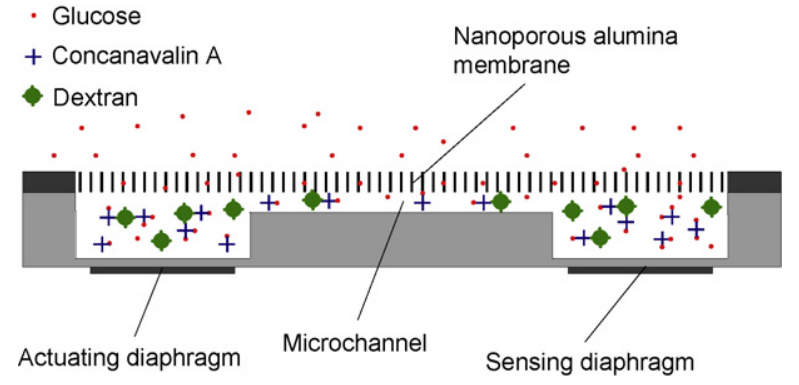

Fig. 1. Schematic illustration of the nanoporous alumina membrane in the glucose affinity sensor. The large sensing molecules (ConA and dextran) are confined in the sensor whilst glucose permeates through the membrane.

\section{Experimental}

\subsection{Diffusion cells}

The ConA retention and glucose diffusion properties of $2-4 \mathrm{~nm}$ annealed nanoporous alumina membranes from Synkera Technologies were assessed using home-made diffusion cells fabricated in PMMA (poly(methyl methacrylate)) (Fig. 2). The two diffusion cells had a volume of $140 \mu \mathrm{l}$ with a diffusing surface of about $28 \mathrm{~mm}^{2}$. The two chambers were separated by the nanoporous membrane, which was glued using medical-grade adhesive epoxy (Loctite M$21 \mathrm{HP}$ ) for ensuring leak-tight measurements. First, a solution with a given concentration of the solute was injected in the first cell whilst pure solvent was injected in the second one. Both cells could be stirred by standard PTFE (Teflon) magnetic stirrers. The solute diffusion was monitored by measuring the solute concentration by either refractometry or UV absorbance spectrometry.

\subsection{Refractometry}

The glucose concentration was determined by measuring the refractive index of the solution using a high precision refractometer (Bellingham and Stanley RFM 342). A volume as small as $60 \mu \mathrm{l}$ is required for refractive index measurements carried out at the reference wavelength of $589.3 \mathrm{~nm}$ (the sodium D line). The refractive index depends almost linearly on glucose concentration. An accuracy of \pm 0.00004 is reported, which corresponds to $1.5 \mathrm{mM}$ of glucose concentration.

For determining the diffusion of glucose molecules, the diffusion cells were respectively filled with a $100 \mathrm{mM}$ glucose solution and ultrapure water. Before each experiment, a two point calibration was done. The refractive index values were then converted into glucose concentrations using the calibration curve.

The effective diffusion coefficient was extracted from the time dependent glucose concentration assuming that a steady-state concentration profile is always satisfied within the membrane [13]. Under these assumptions, combining the diffusion equation with

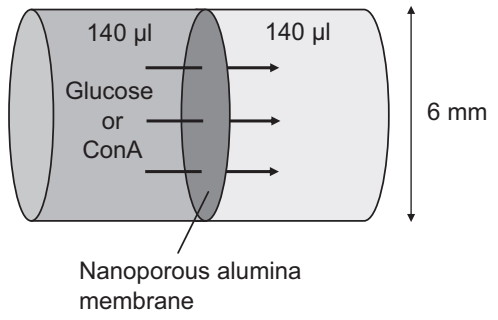

Fig. 2. Glucose diffusion and ConA retention were evaluated using diffusion cells. The concentration of permeating molecules was monitored by refractometry or UV absorbance spectrometry during tests.
Fick's law and mass balance conditions between the two chambers gives

$C_{2}-C_{1}=\left(C_{2,0}-C_{1,0}\right) \exp \left(\frac{-t}{\tau}\right)$

with

$\tau=\frac{l}{D_{e f f} S} \frac{V_{1} V_{2}}{V_{1}+V_{2}}$

where $C_{i}$ and $V_{i}$ are respectively the concentration and volume of the chamber $i, l$ is the membrane thickness, $S$ is the membrane area, and $D_{\text {eff }}$ is the effective diffusion constant in the membrane. From this relationship, we expect that the solution concentrations across the porous membrane will vary exponentially with time.

\subsection{UV absorbance spectrometry}

UV absorbance spectrometry was used to quantify the concentration of ConA in solution. The tyrosine $(274 \mathrm{~nm})$ and tryptophan $(280 \mathrm{~nm})$ aromatic amino acids were detected as they are the most absorbing components of proteins. The UV light was transmitted via an optical fiber from the UV light source (DT-MINI-2-GS from Ocean Optics), then passed through the sample contained in a micro-cuvette $(70 \mu \mathrm{l})$, and was collected by a spectrometer (USB4000-UV-VIS from Ocean Optics). Based on a calibration done with buffered solutions containing various concentrations of ConA in the range of interest $(0.01-0.1 \%[\mathrm{w} / \mathrm{w}])$, the measured UV intensities were further used to determine the relative absorption induced by ConA.

It is known that ConA is instable in solution and precipitates after a few hours. As its solubility is strongly increased in presence of dextran [14], the retention experiments were performed using the sensitive fluid. The sensitive fluid consists in $2 \%[\mathrm{w} / \mathrm{w}] \mathrm{dex}-$ $\operatorname{tran} 3200$ (PSS) and 0.4\% [w/w] ConA (Sigma) in a buffered saline solution ( $10 \mathrm{mM}$ Tris base, $1 \mathrm{mM} \mathrm{CaCl}, 1 \mathrm{mM} \mathrm{MnCl}_{2}, 0.05 \% \mathrm{NaN}_{3}$, $0.15 \mathrm{M} \mathrm{NaCl}$ ). The two diffusion cells were respectively filled with the sensitive fluid and an isotonic saline solution (the same solution but without dextran and ConA). The glucose concentration of both solutions was $30 \mathrm{mM}$, a relatively high concentration where most ConA molecules are in their unbounded state, which favours their diffusion through the nanoporous membrane. For determining the ConA diffusion coefficient, a sample of isotonic saline solution was removed from the diffusion cell and placed in the micro-cuvette for recording the UV absorption spectrum. The ConA retention was evaluated by measuring its concentration in the isotonic saline solution every $24 \mathrm{~h}$, after what the solution was replaced by a fresh one.

\subsection{Nanoporous membrane in a glucose sensor}

To evaluate the long term membrane functionality, a sensor was fabricated as described in Boss et al. [12]. Basically, the sensor includes both an actuating and a sensing piezoelectric diaphragms as well as a flow-resistive microchannel used for viscosity detection. The sensor was filled with the sensitive fluid further confined with a 2-4 nm nanoporous alumina membrane from Synkera Technologies. We used a low viscosity sensitive fluid prepared following Kuenzi et al. recipes [15]. The sensor response was evaluated by performing continuous glucose measurements in isotonic saline solution at $37^{\circ} \mathrm{C}$. Glucose solutions were pumped into the test cell using a computer controlled syringe pump (Tecan CavroXCalibur). 


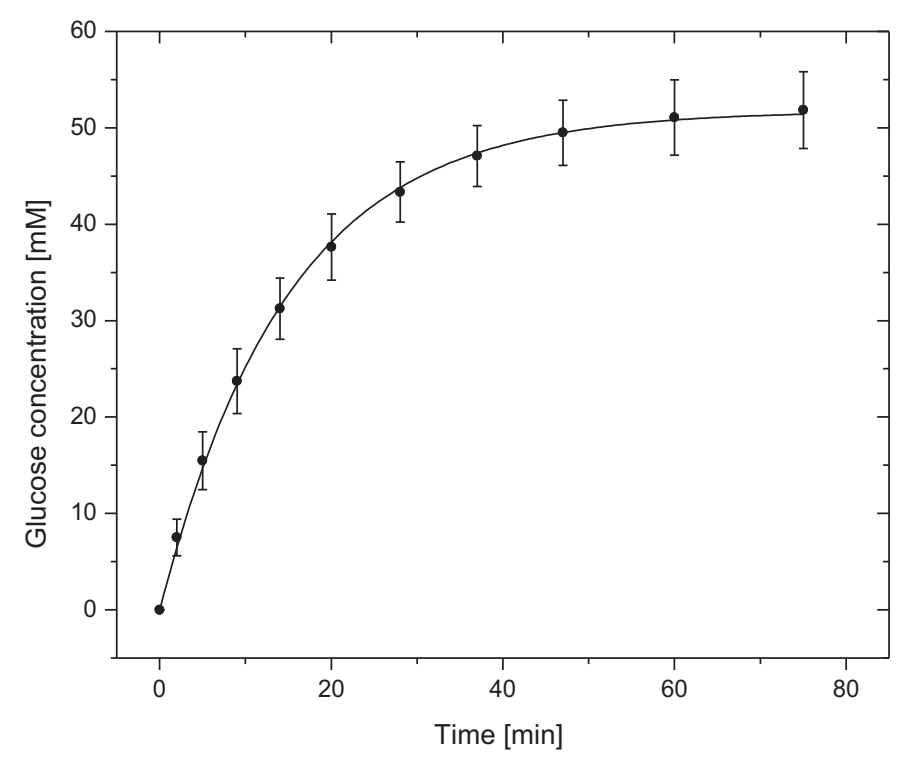

Fig. 3. Dynamics of glucose diffusion through 5 different $2-4 \mathrm{~nm}$ nanoporous alumina membranes fitted by an exponential law.

\section{Results and discussion}

\subsection{Glucose diffusion}

The glucose diffusion properties of $2-4 \mathrm{~nm}$ nanoporous alumina membranes were characterized by measuring the glucose diffusion in 5 different membranes (Fig. 3). As expected from the diffusion cells model, the dynamics of glucose diffusion is pretty well fitted by an exponential law. The relaxation time determined by best fitting gives a mean relaxation time of $15.3 \pm 3.5 \mathrm{~min}$. The effective diffusion coefficients were calculated from the relaxation time following Eq. (1), which gives $(1.35 \pm 0.31) \times 10^{-4} \mathrm{~mm}^{2} / \mathrm{s}$. From these results, it comes out that the glucose diffusion coefficient in these membranes is only $\sim 5$ times smaller than in water $\left(D=6.73 \times 10^{-4} \mathrm{~mm}^{2} / \mathrm{s}[16]\right)$. Considering the relative standard deviation of $23 \%$, this value is of the same order of magnitude that the diffusion coefficient in a $20 \mathrm{~nm}$ asymmetric alumina membrane from Whatman $\left(1.39 \times 10^{-4} \mathrm{~mm}^{2} / \mathrm{s}\right)$ and much higher than its value in nanoporous polyethylene membrane $\left(0.18 \times 10^{-4} \mathrm{~mm}^{2} / \mathrm{s}\right)$ [2]. This quite high diffusivity is remarkable when considering all possible effects impeding diffusion in nanoporous membranes. One important factor is the membrane porosity, which strongly reduces the active diffusing surface in nanoporous membranes. From SEM pictures (Fig. 6), the porosity of the active layer with $13 \pm 2 \mathrm{~nm}$ alumina membranes was estimated to be $28.9 \%$, which explains most of the reduction in diffusion. In addition, when moving to nanofiltration application, the free diffusion is progressively restricted when the dimensions of the pores become comparable with those of the diffusing molecules. Factors explaining this limited diffusion may include an increased viscous drag in the pore, particle-pore wall interactions, as well as steric effects at the entrance of the pore [17]. With a factor of about 4 between the glucose hydrodynamic radius $(0.365 \mathrm{~nm}$ [18]) and the pore size, we are typically in a situation where the high solute-to-pore ratio may restrict diffusion. This is confirmed by previous results which reported that glucose diffusion was already substantially reduced in a $7 \mathrm{~nm}$ nanoporous silicon nitride membrane [19]. However, from the quite good diffusion properties of the present membrane, it seems that the diffusion is only slightly affected, which is explained by the asymmetrical structure of the membrane. In fact, only a very thin active layer $(0.5-1.5 \mu \mathrm{m})$ restricts diffusion whereas most of the diffusion takes

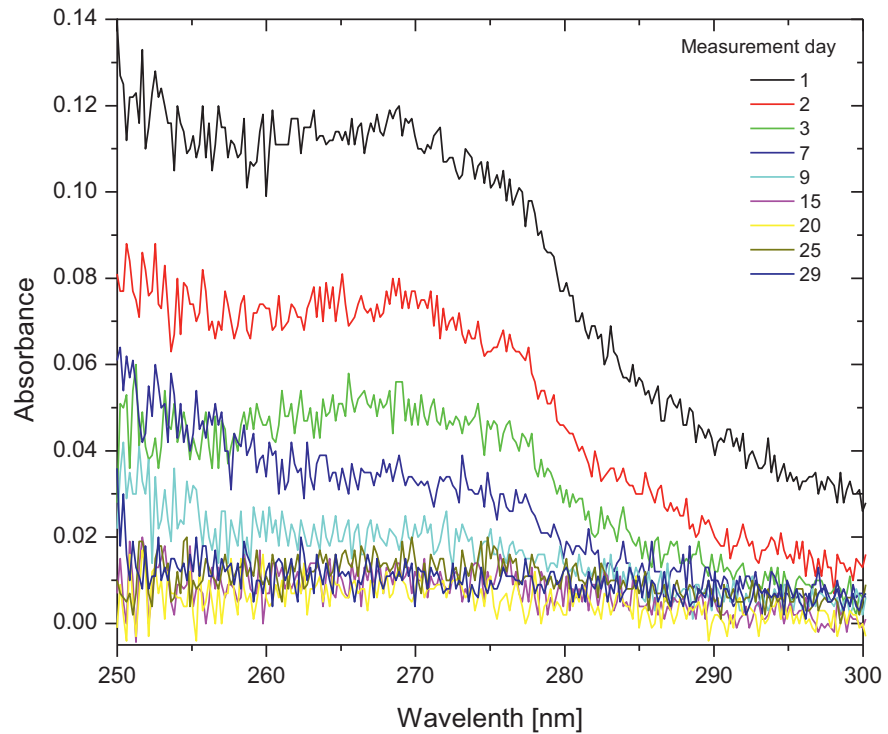

Fig. 4. UV absorbance spectra of the retention test of the $2-4 \mathrm{~nm}$ alumina nanoporous membrane (unstirred solutions). Each spectrum characterizes the ConA permeation during $24 \mathrm{~h}$.

place in the support layer $(50 \mu \mathrm{m})$, which is characterized by larger pores (150-200 nm) which do not impede diffusion. A detailed analysis of the membrane structure would be required to confirm these hypotheses. However, this would require resolving the $2-4 \mathrm{~nm}$ pores, which is hardly achievable by electronic microscopy.

\subsection{Concanavalin A retention}

The ConA retention properties of $2-4 \mathrm{~nm}$ alumina membranes from Synkera Technologies were investigated during 35 days. We recorded the UV absorbance spectrum of the isotonic saline solution which was in contact with the membrane during $24 \mathrm{~h}$ (Fig. 4). A stronger absorbance is observed around a wavelength of $280 \mathrm{~nm}$, indicating the presence of ConA. From these results, the ConA concentration was determined by averaging the absorbance over a $10 \mathrm{~nm}$ bandwidth around the peak absorbance. The error on the ConA concentration was estimated from the standard deviation of the mean absorbance and background noise. ConA leakage was finally expressed in terms of percentage of the ConA concentration in the sensitive fluid (Fig. 5). We performed ConA retention experiments with both well-stirred and unstirred solutions to confirm that stirring had no deleterious effect on ConA molecules (which could have eased ConA diffusion). A similar behaviour was observed for both well-stirred and unstirred solutions, which is explained by the constant quasi-equilibrium of the solutions (diffusion of a very small fraction of ConA) and the long time scale allowing diffusion in the cells. Therefore both retention experiments can be used to estimate the retention capabilities of the membrane.

The ConA retention experiments showed a higher initial ConA leakage just after the beginning of the experiment. Then, after about 5-10 days the leakage stabilized at a lower level. In particular, ConA leakage was well described by an exponential model reaching a constant value. Initial higher leakage could be attributed to ConA fragments which are present in commercial ConA [20]. These fragments do not form tetramer and have a molecular weight 2-3 times smaller than the unaltered subunit. Due to their smaller size, ConA fragments could diffuse through the nanoporous membrane. After that initial ConA leakage, a lower and stable value was observed with a mean leakage per day of $0.14 \pm 0.07 \%$ of the initial concentration. As the measured ConA concentrations were small $( \pm 0.001 \%[\mathrm{w} / \mathrm{w}])$ and close to the resolution of the UV 


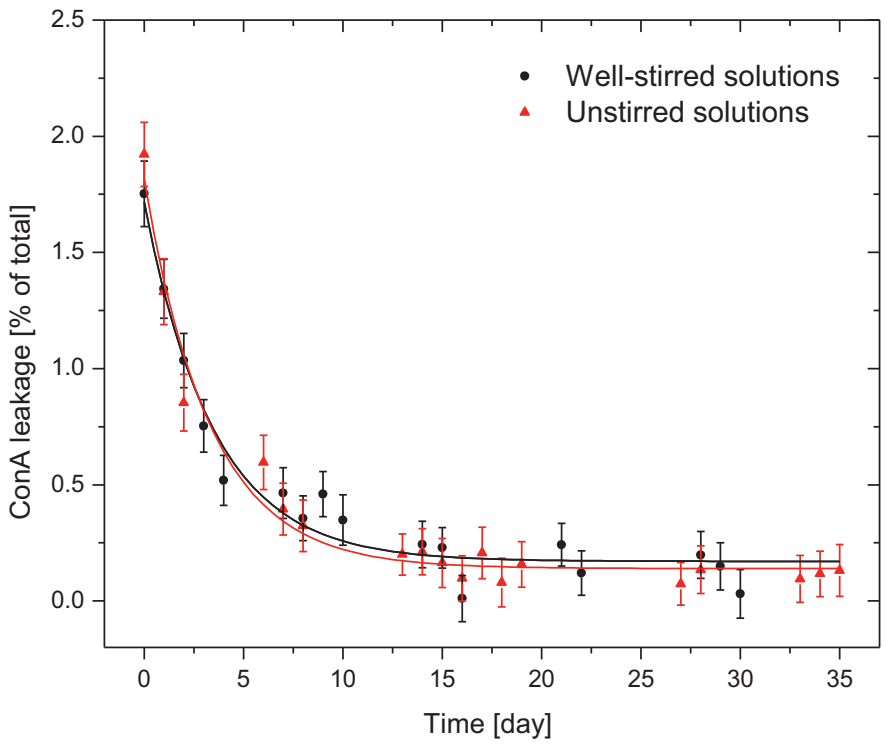

Fig. 5. Long term ConA retention of two different $2-4 \mathrm{~nm}$ nanoporous alumina membranes fitted with an exponential law. ConA leakage is expressed in terms of percentage of the ConA concentration in the sensitive fluid.

absorbance spectrometer, the error on the determination of ConA leakage was quite high. However, ConA leakage is in the same range for both experiments, thus validating the experimental procedure despite the limited accuracy.

An almost complete retention was achieved by the membrane, which has to be emphasized given that ConA is a small protein. At $\mathrm{pH} 7.4$, most of ConA is in tetramer configuration (hydrodynamic radius of $4.4 \mathrm{~nm}$ ) although some dimmers remain (hydrodynamic radius of $3.3 \mathrm{~nm}$ ) [21,22]. According to similar dimensions between the ConA molecules and the membrane pore size, we could have expected a complete retention. The small remaining diffusion can be explained by a pore size distribution not as narrow as expected, some fraction being above the specifications. Similarly, whereas ConA tetramers should be well confined by the membrane, the small fraction of dimmers could still freely permeate through the membrane. Nevertheless, the very small diffusion rate suggests that the pores are achieving an almost complete retention.

\subsection{Pore size distribution}

The nanoporous alumina membrane structure was investigated by scanning electron microscopy (SEM) (Fig. 6). These pictures confirm the highly ordered structure and well defined pore size that is expected for nanoporous alumina membranes. The $2-4 \mathrm{~nm}$ pores were close to the resolution of the microscope and thus we were not able to get well focused pictures. We therefore investigated the pore size distribution on a similar alumina membrane with larger pores ( $13 \pm 2 \mathrm{~nm}$ from Synkera Technologies). The pore size distribution was experimentally determined using particle analysis. An area encompassing 2583 pores was measured on 5 SEM pictures (Fig. 7). The pore size distribution is well described by a Gaussian with a mean pores diameter of $16.49 \mathrm{~nm}$ and a standard deviation of $4.93 \mathrm{~nm}$, confirming a regular pore size distribution. The mean porosity is $28.9 \pm 0.7 \%$, which can be compared to the $37 \%$ reported for Whatman alumina membranes having a nominal pore size of $20 \mathrm{~nm}$ [23]. These measurements confirm that these alumina nanoporous membranes have a distribution not as narrow as expected, and as a consequence, a small fraction of pores is outside the main distribution with dimensions as large as $30 \mathrm{~nm}$. The pore size distribution is also probably larger than expected for
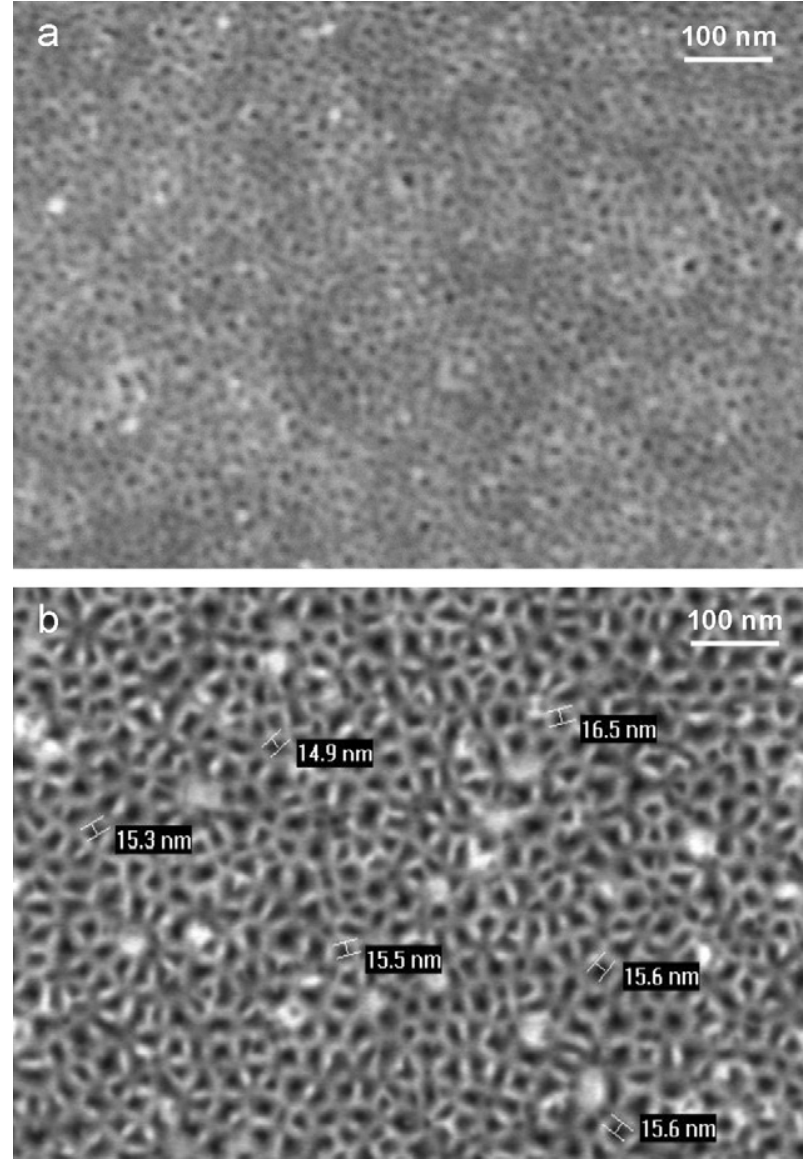

Fig. 6. SEM pictures showing the active layer pore size of the nanoporous alumina membranes. (a) 2-4 $\mathrm{nm}$ membrane. (b) $13 \pm 2 \mathrm{~nm}$ membrane. (Image pixel size of $1.1 \mathrm{~nm}$.)

the 2-4 nm alumina membrane, which may explain the small ConA diffusion at large time scales.

\subsection{Membrane performance in a glucose sensor}

The long term performance of a nanoporous alumina membrane as size-selective interface was evaluated by continuously

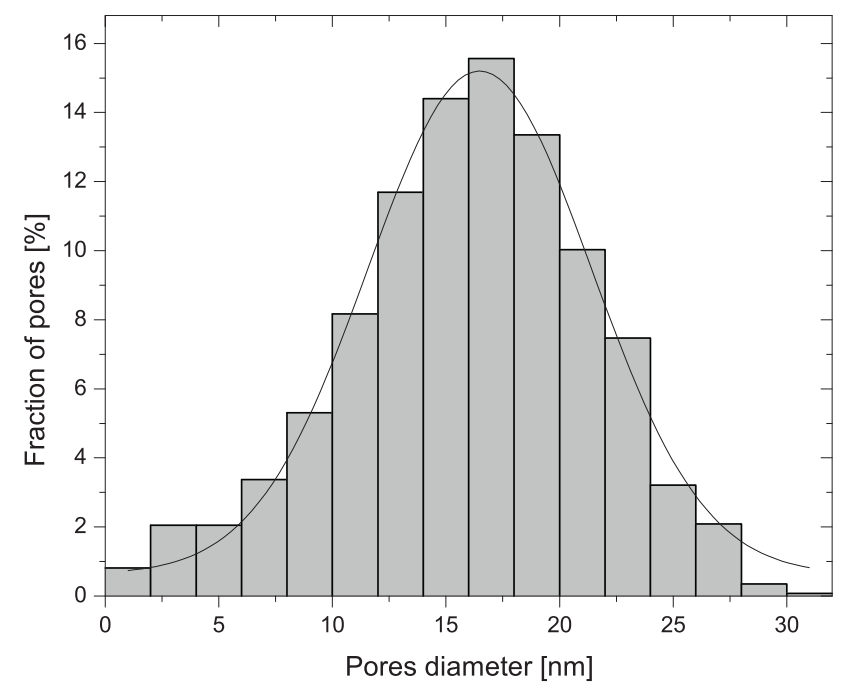

Fig. 7. Experimental characterization (SEM pictures) of the pore size distribution of the $13 \pm 2 \mathrm{~nm}$ membrane fitted with a Gaussian. 


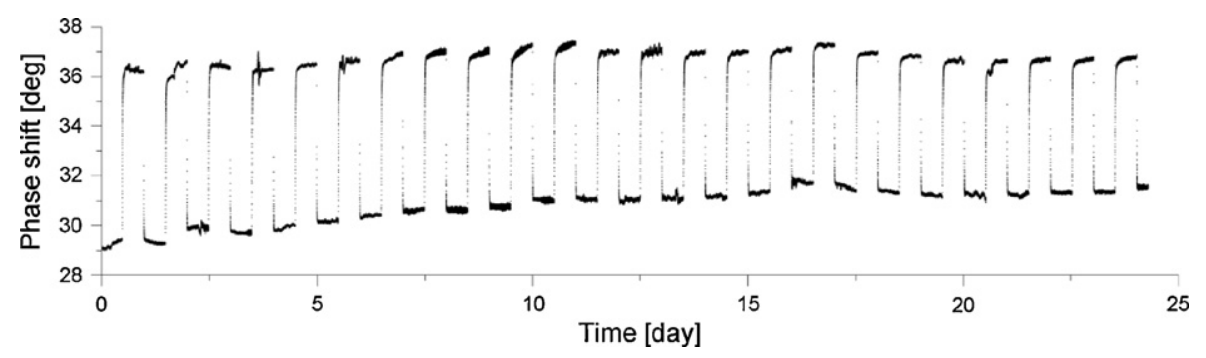

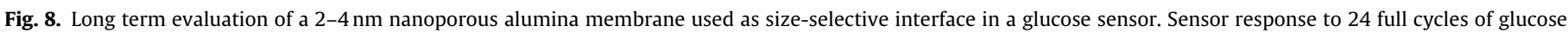
concentrations ( $2 \mathrm{mM}$ and $12 \mathrm{mM}$ ) in isotonic saline solution at $37^{\circ} \mathrm{C}$.

measuring the glucose concentration in isotonic saline solution during 25 days (Fig. 8). The sensor was tested in the most relevant physiologically glucose concentrations, namely $2 \mathrm{mM}$ and $12 \mathrm{mM}$, at physiological temperature $\left(37^{\circ} \mathrm{C}\right)$. The sensor worked well during the whole experiment, glucose diffusing freely in and out of the sensor through the nanoporous membrane, whereas the sensitive fluid was still confined inside the sensor. This good behaviour throughout the experiment also demonstrates the excellent long term stability of the membrane in aqueous solution at $37^{\circ} \mathrm{C}$. Moreover, the response time of the sensor did not increase with time, indicating neither dextran nor ConA clogged the pores. Besides these promising characteristics, the sensor sensitivity decreased linearly with a reduction of $1.0 \% /$ day. The small ConA diffusion observed in the retention experiments could partly explain this reduction in sensitivity. ConA deactivation could also be involved in the sensitivity reduction, as suggested by other studies reporting long term stability of ConA-based sensors [10,11]. These results confirm that nanoporous alumina membranes are promising for selective interface in affinity sensors. Moreover, membrane manufacturing companies like Synkera Technologies are actively working on achieving precise pore size control below $2 \mathrm{~nm}$. We could therefore expect much better long term stability for next generation of nanofiltration membranes.

\section{Conclusion}

The diffusion, retention and pore size distribution of $2-4 \mathrm{~nm}$ nanoporous alumina membranes were characterized in details. The very thin active layer of the membrane $(0.5-1.5 \mu \mathrm{m})$ and quite high porosity (28.9\%) gives remarkable diffusion properties, with only a 5 -fold reduction compared to free diffusion in water. We also report an almost complete retention of Concanavalin A (hydrodynamic radius of $3.3 \mathrm{~nm}$ ) and a good stability in aqueous solution up to 35 days. SEM pictures also confirm the highly ordered structure and well defined pore size that is expected for nanoporous alumina membranes. Finally, the nanoporous alumina membrane was successfully used as dialysis membrane in an affinity glucose sensor during 25 days without any biofouling. These results suggest that nanoporous alumina membranes could be used as size-selective interface on biosensors.

\section{References}

[1] R.F. Turner, D.J. Harrison, R.V. Rajotte, Preliminary in vivo biocompatibility studies on perfluorosulphonic acid polymer membranes for biosensor applications, Biomaterials 12 (1991) 361-368.
[2] H. Uehara, M. Kakiage, M. Sekiya, D. Sakuma, T. Yamonobe, N. Takano, A. Barraud, E. Meurville, P. Ryser, Size-selective diffusion in nanoporous but flexible membranes for glucose sensors, ACS Nano 3 (2009) 924-932.

[3] T.A. Desai, D.J. Hansford, L. Leoni, M. Essenpreis, M. Ferrari, Nanoporous antifouling silicon membranes for biosensor applications, Biosens. Bioelectron. 15 (2000) 453-462.

[4] W. Lee, R. Ji, U. Gosele, K. Nielsch, Fast fabrication of long-range ordered porous alumina membranes by hard-anodization, Nat. Mater. 5 (2006) 741-747.

[5] C.J. Ingham, J. ter Maat, W.M. de Vos, Where bio meets nano: the many uses for nanoporous aluminum oxide in biotechnology, Biotechnol. Adv. (2011), doi:10.1016/j.biotechadv.2011.08.005.

[6] D. Gong, V. Yadavalli, M. Paulose, M. Pishko, C.A. Grimes, Controlled molecular release using nanoporous alumina capsules, Biomed. Microdevices 5 (2003) $75-80$.

[7] K.E. La Flamme, G. Mor, D. Gong, T. La Tempa, V.A. Fusaro, C.A. Grimes, T.A. Desai, Nanoporous alumina capsules for cellular macroencapsulation: transport and biocompatibility, Diabetes Technol. 7 (2005) 684-694.

[8] O. Krushinitskaya, T.I. Tonnessen, H. Jakobsen, E.A. Johannessen, Characterization of nanoporous membranes for implementation in an osmotic glucose sensor based on the concanavalin A - dextran affinity assay, J. Membr. Sci. 376 (2011) 153-161.

[9] J. Wang, Electrochemical glucose biosensors, Chem. Rev. 108 (2008) 814-825.

[10] R. Ballerstadt, A. Polak, A. Beuhler, J. Frye, In vitro long-term performance study of a near-infrared fluorescence affinity sensor for glucose monitoring, Biosens. Bioelectron. 19 (2004) 905-914.

[11] R. Ballerstadt, J.S. Schultz, A fluorescence affinity hollow fiber sensor for continuous transdermal glucose monitoring, Anal. Chem. 72 (2000) 4185-4192.

[12] C. Boss, E. Meurville, J.-M. Sallese, P. Ryser, A viscosity-dependent affinity sensor for continuous monitoring of glucose in biological fluids, Biosens. Bioelectron. 30 (2011) 223-228.

[13] E.L. Cussler, Diffusion, Mass Transfer in Fluidic Systems, third ed., Cambridge University Press, Cambridge, 2009.

[14] J.J. Kim, K. Park, Glucose-binding property of pegylated concanavalin A, Pharm. Res. 18 (2001) 794-799.

[15] S. Kuenzi, E. Meurville, P. Ryser, Automated characterization of dextran/concanavalin A mixtures-a study of sensitivity and temperature dependence at low viscosity as basis for an implantable glucose sensor, Sens. Actuators B: Chem. 146 (2010) 1-7.

[16] L.G. Longsworth, Diffusion measurements, at $25^{\circ}$, of aqueous solutions of amino acids, peptides and sugars, J. Am. Chem. Soc. 75 (1953) 5705-5709.

[17] J.R. Pappenheimer, E.M. Renkin, L.M. Borrero, Filtration, diffusion and molecular sieving through peripheral capillary membranes; a contribution to the pore theory of capillary permeability, Am. J. Physiol. 167 (1951) 13-46.

[18] A. Bouchoux, H. Roux-de Balmann, F. Lutin, Nanofiltration of glucose and sodium lactate solutions: variations of retention between single- and mixedsolute solutions, J. Membr. Sci. 258 (2005) 123-132.

[19] L. Leoni, A. Boiarski, T.A. Desai, Characterization of nanoporous membranes for immunoisolation: diffusion properties and tissue effects, Biomed. Microdevices 4 (2002) 131-139.

[20] J.L. Wang, B.A. Cunningham, G.M. Edelman, Unusual fragments in the subunit structure of concanavalin A, Proc. Natl. Acad. Sci. U.S.A. 68 (1971) 1130-1134.

[21] M. Huet, Factors affecting the molecular structure and the agglutinating ability of concanavalin A and other lectins, Eur. J. Biochem. 59 (1975) 627-632.

[22] G.N. Reeke, J.W. Becker, G.M. Edelman, The covalent and three-dimensional structure of concanavalin A. IV. Atomic coordinates, hydrogen bonding, and quaternary structure, J. Biol. Chem. 250 (1975) 1525-1547.

[23] S.W. Lee, H. Shang, R.T. Haasch, V. Petrova, G.U. Lee, Transport and functional behaviour of poly(ethylene glycol)-modified nanoporous alumina membranes, Nanotechnology 16 (2005) 1335-1340. 